\title{
Strukturbiologie
}

\section{Mitochondrien: wie die Gene im Kraftwerk der Zelle aktiviert werden}

\author{
ARJUN BHATTA ${ }^{1,2}$, HAUKE S. HILLEN ${ }^{1,2,3}$ \\ ${ }^{1}$ INSTITUT FÜR ZELLBIOCHEMIE, UNIVERSITÄTSMEDIZIN GÖTTINGEN, \\ UNIVERSITÄT GÖTTINGEN \\ 2 FORSCHUNGSGRUPPE „STRUKTUR UND FUNKTION MOLEKULARER MASCHINEN“, \\ MPI FÜR MULTIDISZIPLINÄRE NATURWISSENSCHAFTEN, GÖTTINGEN \\ ${ }^{3}$ EXZELLENZCLUSTER „MULTISCALE BIOIMAGING: FROM MOLECULAR MACHINES \\ TO NETWORKS OF EXCITABLE CELLS“ (MBEXC), UNIVERSITÄT GÖTTINGEN
}

Mitochondria contain an organellar genome that encodes for subunits of the respiratory chain. Its coordinated expression is essential for eukaryotic life and defects in this process lead to severe disease. However, the molecular mechanisms underlying mitochondrial gene expression remain poorly understood. Recent structural and functional studies have provided the first mechanistic insights into mitochondrial gene expression and highlight the evolutionarily unique nature of this system.

DOI: $10.1007 / \mathrm{s} 12268-022-1711-\mathrm{Z}$

(C) Die Autoren 2022

Menschliche Zellen enthalten zwei Genome. Zusätzlich zum Erbgut im Zellkern existiert ein zweiter Satz genetischen Materials in den Mitochondrien (mtDNA). Diese Zellorganellen werden häufig als „Kraftwerke der Zelle“ bezeichnet, denn in ihnen wird

mithilfe der Atmungskette Adenosintriphosphat (ATP) gewonnen. Das mitochondriale Genom ist ein evolutionäres Überbleibsel der bakteriellen Urahnen von Mitochondrien und codiert für essenzielle Untereinheiten der Atmungskette. Diese Untereinheiten müssen

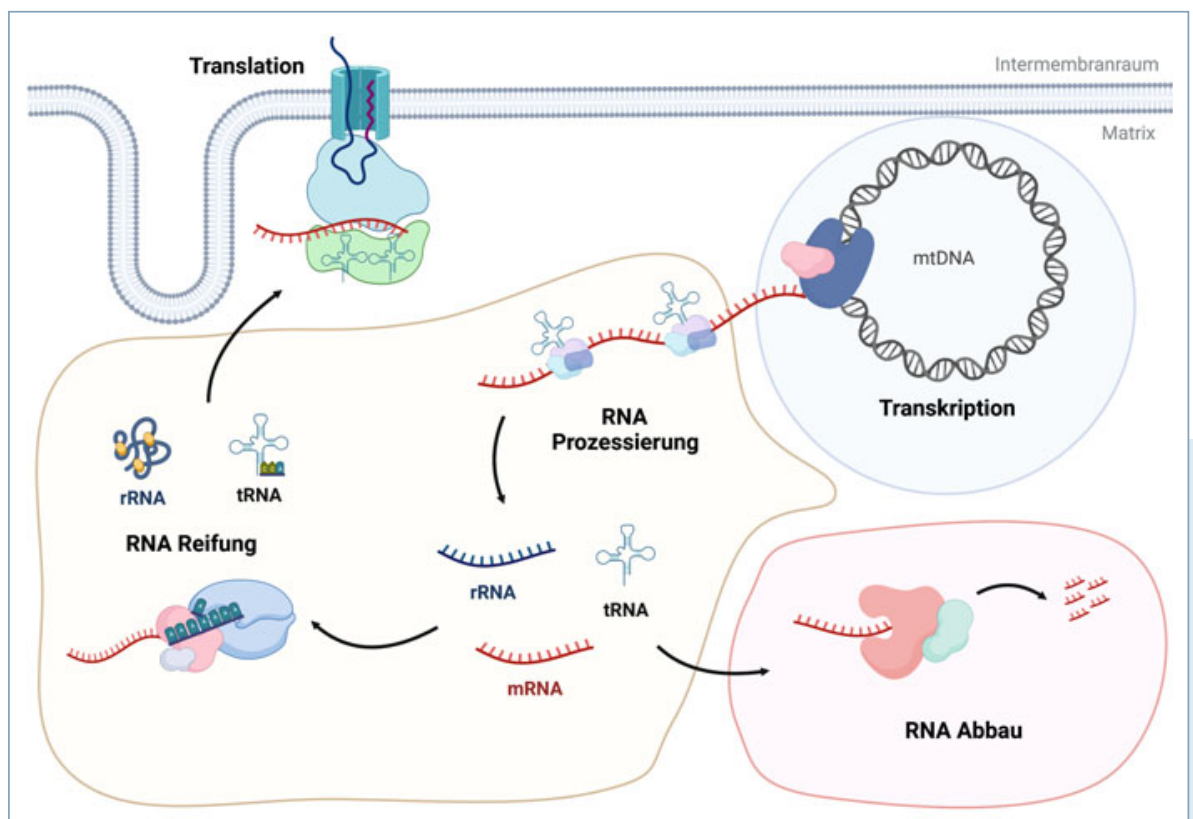

sich mit einer Vielzahl anderer, im Zellkern codierter Untereinheiten zusammenlagern, um funktionsfähige Komplexe zu bilden. Die geregelte Expression der mitochondrialen Gene ist daher lebensnotwendig und erbliche Störungen dieses Prozesses führen zu schweren metabolischen Erkrankungen. Die mitochondriale Genexpression wird von spezialisierten molekularen Maschinen ausgeführt, die sich grundlegend von denen im Zellkern oder in Bakterien unterscheiden. So verfügen Mitochondrien über eine dedizierte RNAPolymerase (POLRMT) sowie ein spezialisiertes Ribosom (Mitoribosom). Zwischen Transkription und Translation durchlaufen mitochondriale Transkripte eine Reihe von RNAReifungsschritten, welche vermutlich die Hauptregulierungspunkte der mitochondrialen Genexpression sind (Abb. 1, [1-4]). In den letzten Jahren haben strukturelle und biochemische Arbeiten erste mechanistische Einblicke in die mitochondriale Genexpression geliefert, doch es bleiben noch viele ungelöste Rätsel.

\section{Mitochondriale Transkription}

Der erste Schritt der mitochondrialen Genexpression ist die Transkription. Überraschenderweise ist die mitochondriale RNAPolymerase weder mit anderen zellulären noch mit bakteriellen RNA-Polymerasen verwandt, sondern ähnelt den RNA-Polymerasen von Bakteriophagen [5]. Im Gegensatz $\mathrm{zu}$ diesen benötigt POLRMT allerdings zusätzliche Proteinfaktoren für die Transkription. Diese mitochondrialen Transkriptionsfaktoren zeigen keinerlei Sequenzhomologie zu Transkriptionsfaktoren im Zellkern

4 Abb. 1: Schematischer Überblick der Genexpression in menschlichen Mitochondrien. Bei der Transkription des zirkulären Genoms entstehen polycistronische Primärtranskripte, die anschließend prozessiert werden müssen, um die verschiedenen RNA-Typen freizusetzen. Diese durchlaufen dann verschiedene weitere Reifungsschritte vor der Translation oder werden wieder abgebaut. Erstellt mit Biorender.com, verändert mach [12]. 


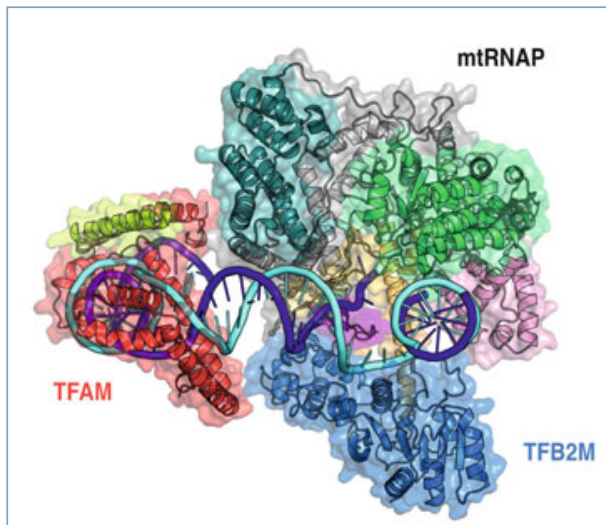

Initiationskomplex

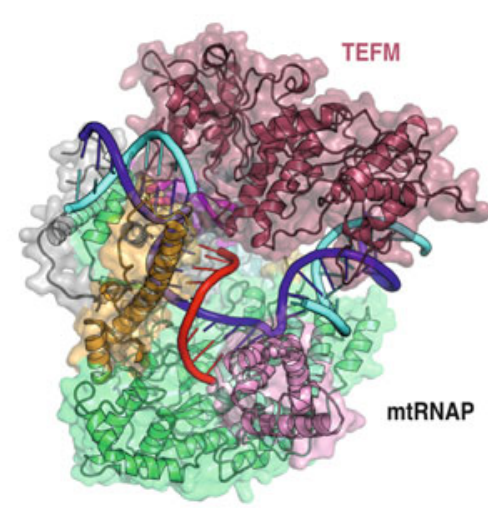

Elongationskomplex mit TEFM

$\triangle$ Abb. 2: Strukturen mitochondrialer Transkriptionskomplexe. Links: mitochondrialer Initiationskomplex (PDB: 6ERP) [6]. Rechts: Struktur des mitochondrialen Elongationskomplexes mit Elongationsfaktor TEFM (PDB: 5OLA) [7].

oder in Bakterien, sodass ihre Funktionsweise lange Zeit unklar blieb. Mithilfe der Strukturbiologie konnten wir dieses Rätsel lösen, indem wir Momentaufnahmen der mitochondrialen RNA-Polymerase in funktionalen Komplexen mit verschiedenen Transkriptionsfaktoren lösten (Abb. 2). So konnten wir zum einen die Struktur des Initiationskomplexes aufklären, welcher aus POLRMT, DNA und den beiden Initiationsfaktoren TFAM und TFB2M besteht [6]. Diese zeigt, dass TFAM die Polymerase zum Promoter rekrutiert, während TFB2M einen Einzelstrang der aufgeschmolzenen DNA bindet und so den Initiationskomplex stabilisiert. Zum anderen konnten wir zeigen, wie der Elongationsfaktor TEFM an die transkribierende POLRMT bindet [7]. Dieser Faktor stimuliert die Transkription, indem er die aufgeschmolzene DNA in der Transkriptionsblase stabilisiert und mit POLRMT einen Tunnel bildet, durch den die naszente RNA aus dem aktiven Zentrum geführt wird. Letzteres verhindert vermutlich die Bildung von Sekundärstrukturen in der RNA und verhindert so frühzeitige Kettenabbrüche. In Kombination mit vorherigen Studien ergibt sich aus diesen Strukturen ein molekulares Bild des Transkriptionszyklus in menschlichen Mitochondrien [8].

Die Strukturen zeigen zudem einen interessanten Aspekt der mitochondrialen Genexpression auf, denn sowohl TFB2M als auch TEFM ähneln strukturell Proteinen, die an völlig anderen Prozessen beteiligt sind. So weist TFB2M eine Methyltransferase-Faltung auf, während TEFM einer Holliday-JunctionResolvase gleicht. In beiden Fällen werden die Nukleinsäure-bindenden Eigenschaften dieser Proteinfamilien für eine Funktion in der mitochondrialen Transkription genutzt.

\section{Inhibition der mitochondrialen Transkription als neue Therapiemöglichkeit}

Die molekularen Erkenntnisse über die Mechanismen der mitochondrialen Transkription bergen möglicherweise auch thera- peutisches Potenzial. So konnte ein Forschungsteam unter Federführung unserer Kollaborationspartner Nils-Göran Larsson (Karolinska Institutet, Schweden) und Nina Bonekamp (Universitätmedizin Mannheim) kürzlich eine neue Klasse von Molekülen entwickeln, welche hochspezifisch die POLRMT inhibieren (IMTs). In vitro- und in vivo-Versuche mit diesen Inhibitoren zeigen, dass die Hemmung der mitochondrialen Transkription eine therapeutische Strategie zur Behandlung von Krebserkrankungen darstellen könnte [9]. Mithilfe der Kryoelektronenmikroskopie (Kryo-EM) konnten wir zeigen, dass IMTs hochspezifisch in eine hydrophobe Bindungstasche nahe dem aktiven Zentrum der POLRMT binden und das Enzym so in einer inaktiven Konformation stabilisieren, die keine Nukleinsäurebindung zulässt [9]. Dies zeigt, dass IMTs als allosterische Inhibitoren wirken und erklärt, weshalb sie spezifisch nur die mitochondriale Transkription hemmen.

\section{Mitochondriale RNA-Prozessierung}

Der zweite Schritt der mitochondrialen Genexpression ist die RNA-Prozessierung. Die Transkription des mitochondrialen

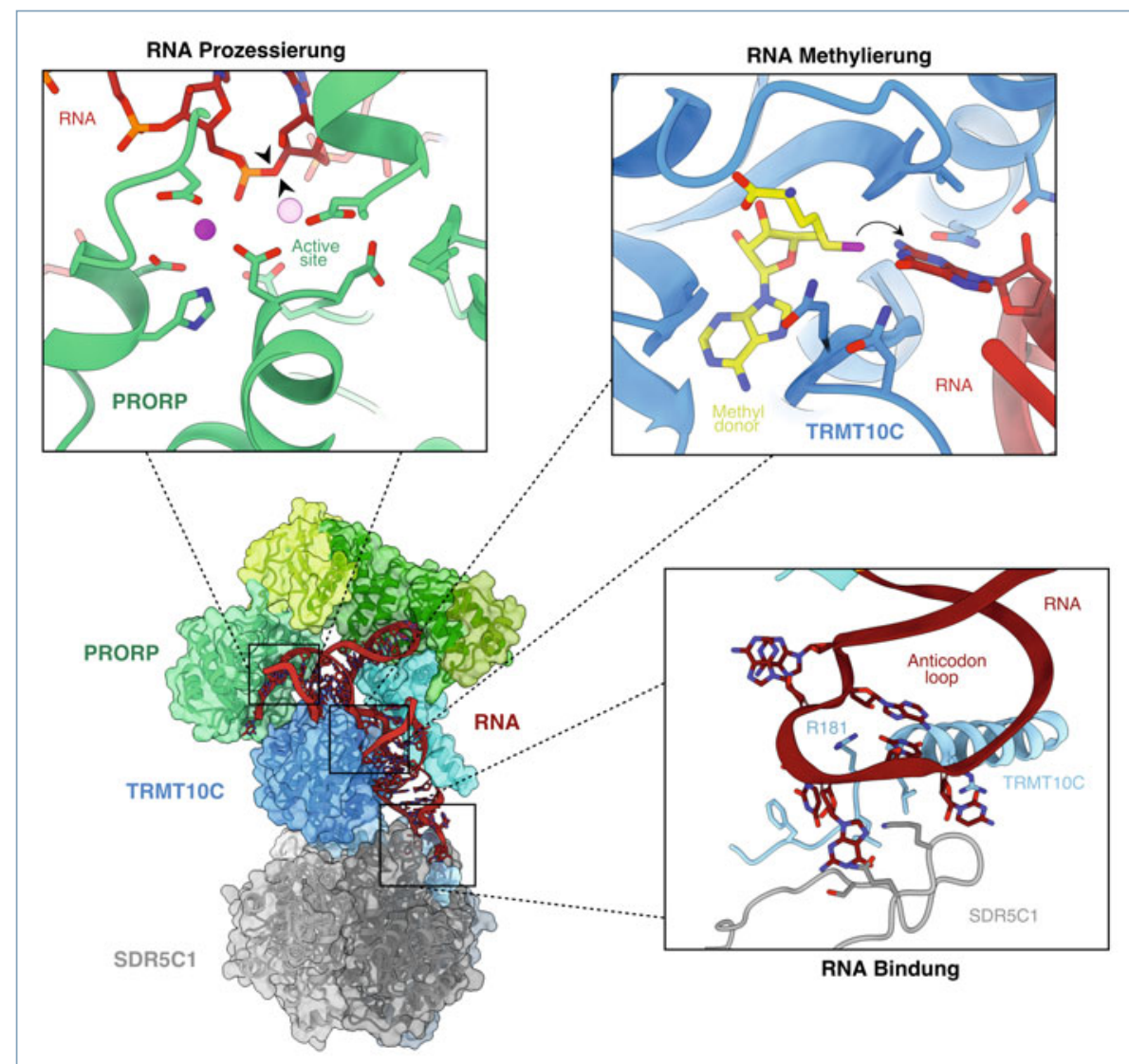

A Abb. 3: Struktur der mitochondrialen RNase P (PDB: 7ONU) [10]. 
Genoms führt zu polycistronischen Primärtranskripten, in denen messenger RNAs (mRNAs) und ribosomale RNAs (rRNAs) jeweils von tRNAs flankiert sind. Um ihrer Funktion nachkommen zu können, müssen die verschiedenen RNA-Abschnitte zunächst getrennt werden. Verantwortlich hierfür sind die mitochondrialen Ribonukleasen (mtRNase) $\mathrm{P}$ und $\mathrm{Z}$, die nacheinander die RNA zunächst am 5“ und dann am 3“ Ende der tRNAs schneiden. Die RNA-Prozessierung ist ein entscheidender Schritt in der mitochondrialen Genexpression, aber ihre molekularen Mechanismen sind bisher kaum erforscht. Wir konnten kürzlich die Kryo-EM Struktur von mtRNase P lösen, was einen ersten molekularen Einblick in diese Prozesse erlaubt (Abb. 2, [10]). Im Gegensatz zu den RNase-P-Enzymen im Zellkern oder in Bakterien ist mtRNase P kein Ribozym, enthält also keine katalytische RNA, sondern besteht stattdessen aus drei Proteinuntereinheiten: SDR5C1, TRMT10C und PRORP. Zudem prozessiert mtRNase P nicht nur die RNAs, sondern methyliert gleichzeitig auch eine Base an Position 9 der tRNA. Während PRORP für die Spaltung der RNA und TRMT10C für deren Methylierung verantwortlich ist, handelt es sich bei SDR5C1 um eine Dehydrogenase, deren Funktion in diesem Komplex bisher rätselhaft war. Die Struktur zeigt, dass SDR5C1 eine strukturelle Funktion übernimmt und mit TRMT10C einen Subkomplex bildet, an den die tRNA über einen ungewöhnlichen Mechanismus bindet. Während andere RNase-P-Enzyme stets den „Ellenbogen“ der tRNA binden, interagiert mtRNase P stattdessen spezifisch mit dem Anticodon-Loop. Dies könnte mit den Sequenzeigenschaften der mitochondrialen tRNAs zusammenhängen, denn die Ellenbogen-Region ist in diesen sehr variabel. Im Gegensatz dazu ist die Base im Anticodon-Loop, die von einem Arginin-Rest in TRTM10C (R181) spezifisch erkannt wird, in allen mitochondrialen tRNAs ein Pyrimidin. Diese Interaktion scheint entscheidend für die Funktion von mtRNase P zu sein, denn Mutationen von R181 wurden als Ursache von mitochondrialen Erkrankungen in Patienten identifiziert.

Die Bindung der tRNA an den SDR5C1TRMT10C-Komplex positioniert die zu methy- lierende Base genau neben der Methyltransferase-Domäne von TRMT10C und ermöglicht die Bindung von PRORP, welches sowohl mit der tRNA als auch mit TRMT10C interagiert, um die Nukleasedomäne genau über der Schnittstelle am 5'-Ende der tRNA zu positionieren. Die Struktur von mtRNase P offenbart somit nicht nur den Mechanismus des ersten Schritts der mitochondrialen RNAProzessierung, sondern zeigt auch ein weiteres Beispiel für die Adaption funktionell nicht verwandter Proteinfamilien für eine Rolle in der mitochondrialen Genexpression auf.

\section{Ausblick}

Durch die Kombination aus genetischen, biochemischen und strukturbiologischen Arbeiten konnten in den letzten Jahren erste mechanistische Einblicke in die mitochondriale Genexpression gewonnen werden. Insbesondere die Mechanismen der Transkription [8] und Translation [11] wurden im Detail untersucht. Im Gegensatz dazu sind die Mechanismen der darauffolgenden RNAProzessierungs- und -Reifungsschritte allerdings weiterhin weitgehend unerforscht. Mit der strukturellen Charakterisierung der mtRNase P haben wir kürzlich erste Einblicke in die mitochondriale RNA-Prozessierung gewinnen können. Darauf aufbauend werden wir in Zukunft mithilfe von modernen strukturbiologischen Methoden, wie der der Kryoelektronenmikroskopie, daran arbeiten, das Puzzle der mitochondrialen Genexpression weiter zu lösen.

\section{Literatur}

[1] Gustafsson CM, Falkenberg M, Larsson N-G (2016) Maintenance and expression of mammalian mitochondrial DNA. Ann Rev Biochem 85: 133-160

[2] Hällberg BM, Larsson N-G (2014) Making proteins in the powerhouse. Cell Metabolism 20: 226-240

[3] Pearce SF, Rebelo-Guiomar P, D'Souza AR et al. (2017) Regulation of mammalian mitochondrial gene expression: recent advances. Trends Biochem Sci 42: 625-639

[4] Barchiesi A, Vascotto C (2019) Transcription, processing, and decay of mitochondrial RNA in health and disease. Int J Mol Sci 20: 2221

[5] Ringel R, Sologub M, Morozov YI et al. (2011) Structure of human mitochondrial RNA polymerase. Nature 478: 269-273 [6] Hillen HS, Morozov YI, Sarfallah A et al. (2017) Structural basis of mitochondrial transcription initiation. Cell 171: 1072-1081.e10

[7] Hillen HS, Parshin AV, Agaronyan K et al. (2017) Mechanism of transcription anti-termination in human mitochondria. Cell 171: 1082-1093.e13

[8] Hillen HS, Temiakov D, Cramer P (2018) Structural basis of mitochondrial transcription. Nat Struct Mol Biol 25: 754-765 [9] Bonekamp NA, Peter B, Hillen HS et al. (2020) Smallmolecule inhibitors of human mitochondrial DNA transcription. Nature 588: 712-716
[10] Bhatta A, Dienemann C, Cramer P, Hillen HS (2021) Structural basis of RNA processing by human mitochondrial RNase P. Nat Struct Mol Biol 28: 713-723

[11] Greber BJ, Ban N (2016) Structure and function of the mitochondrial ribosome. Ann Rev Biochem 85: 103-132 [12] Barchiesi A, Vascotto C (2019) Transcription, Processing, and Decay of Mitochondrial RNA in Health and Disease. int J Mol Sci 20:2221

Funding note: Open Access funding enabled and organized by Projekt DEAL. Open Access: Dieser Artikel wird unter der Creative Commons Namensnennung 4.0 International Lizenz veröffentlicht, welche die Nutzung, Vervielfältigung, Bearbeitung, Verbreitung und Wiedergabe in jeglichem Medium und Forma erlaubt, sofern Sie den/die ursprünglichen Autor(en) und die Quelle ordnungsgemäß nennen, einen Link zur Creative Commons Lizenz beifügen un angeben, ob Anderungen vorgenommen wurden. Die in diesem Artikel
enthaltenen Bilder und sonstiges Drittmaterial unterliegen ebenfalls der

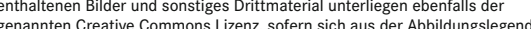
nichts anderes ergibt. Sofern das betreffende Material nicht unter der enannten Creative Commons Lizenz steht und die betreffende Handlung nich nach gesetzlichen Vorschriften erlaubt ist, ist für die oben aufgeführten Weiterverwendungen des Materials die Einwilligung des jeweiligen Rechteinhabers einzuholen. Weitere Details zur Lizenz entnehmen Sie bitte der Lizenzinformation auf http://creativecommons.org/licenses/by/4.0/deed.de.

Korrespondenzadresse:

Prof. Dr. Hauke S. Hillen

Institut für Zellbiochemie

Universitätsmedizin Göttingen

Humboldtallee 23

D-37073 Göttingen

hauke.hillen@med.uni-goettingen.de

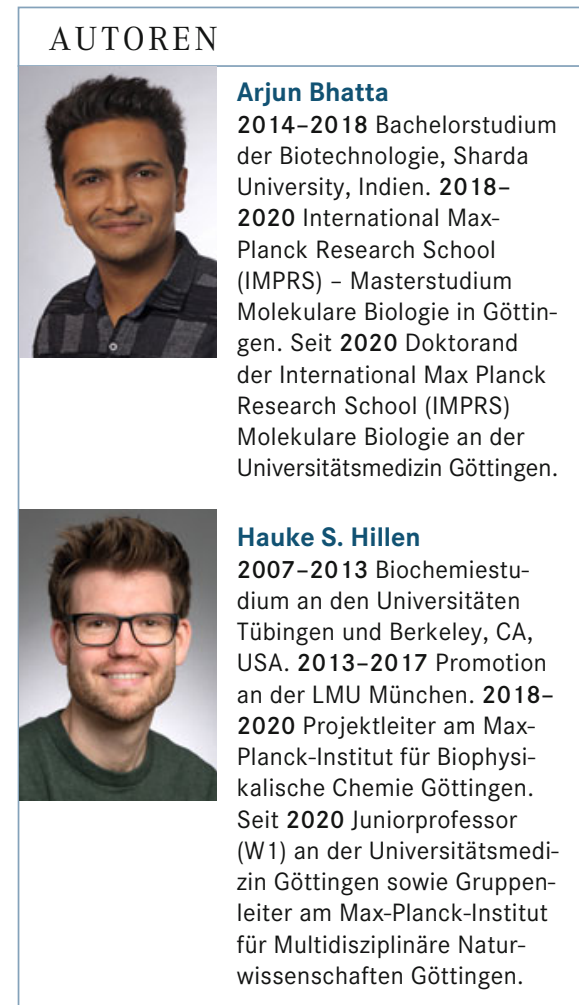

